\title{
SY23-1 Symposium
}

\section{Interprofessional Education: An overview and approaches to IPE in health professions education}

\author{
John L. Szarek
}

Basic Sciences, Geisinger Commonwealth School of Medicine, USA

Emerging evidence regarding the value of healthcare delivered via effective interprofessional teams has created new priorities for interprofessional education (IPE). The WHO in its Framework for Action on Interprofessional Education and Collaborative Practice proposes that an interprofessional collaborative practice-ready healthcare workforce can be achieved through IPE. The WHO defines IPE as occurring "when students from two or more professions learn about, from and with each other to enable effective collaboration and improve health outcomes." Increasingly, health professions schools are placing students together in settings to promote their interprofessional identity. IPE is a global phenomenon. In the United States, the Interprofessional Education Collaborative published core competencies for interprofessional collaborative practice bringing attention to the need for IPE within health professions training in the US. The field has advanced in other global regions as well including, for example, the Centre for the Advancement of Interprofessional Education in the UK, the Australasian Interprofessional Practice and Education Network, the European Interprofessional Practice and Education Network, and the Canadian Interprofessional Health Collaborative. Various models for organizing competencies for interprofessional collaborative practice have been developed to guide development of curricula aimed at student achievement of these competencies. Moreover, accrediting bodies mandate IPE in health professions programs in the US. Integration of IPE into the curricula of health professions schools is challenging. To facilitate integration, one widely used systematic approach to curriculum development is the six-step model. This model provides a framework for developing, implementing, evaluating, and continually improving IPE experiences. Relative to the six steps, the needs for IPE are clear, to enhance patient outcomes and safety. The goals and objectives derived can be aligned with those of professional organizations. Education methods and strategies, and evaluation tools can be developed new or adapted from external resources such as MedEdPORTAL and the National Center for Interprofessional Practice and Education. Finally, faculty may not be prepared to teach in an interprofessional setting, and faculty development thus becomes a necessity. Although challenging, incorporating IPE into our students' education will provide our learners with the training they need to become part of the collaborative practice-ready healthcare workforce. 